\title{
Case Review Two fatal Ehrlichia cases with complete
autopsies
}

\author{
Charles Beavers, Kyle Rickard, James W. Snyder and Sandra
}

C. Hollensead

\author{
Correspondence \\ Charles Beaver; \\ Charles.beavers@louisville.edu
}

Received 10 October 2013

Accepted 30 January 2014

\section{Department of Pathology and Laboratory Medicine, University of Louisville, 530 S. Jackson Street, Louisville, KY 40202, USA}

\begin{abstract}
Introduction: Human monocytic ehrlichiosis (HME) is caused by infection of monocytes by Ehrlichia chaffeensis, an obligate intracellular Gram-negative bacterium. The number of ehrlichiosis cases due to $E$. chaffeensis reported to the Centers for Disease Control and Prevention (CDC) since the disease became reportable in 1999 have increased steadily, from 200 cases in 2000 to 961 cases in 2008. In 2012, the case fatality rate, as reported by the CDC, was $1.1 \%$.
\end{abstract}

Case Presentation: Described in this review are two cases of fatal HME, both of which received a complete autopsy examination. Both cases required serological and molecular studies to reach the final diagnosis. The heart and spleen tissue blocks were diagnostically useful in these cases.

Conclusion: Awareness of HME leads to improved recognition and subsequent timely treatment of this disease. The discovery of HME in our second case was greatly facilitated by the experience gained by the diagnosis in the first case.

Keywords: autopsy; disseminated intravascular coagulation; Ehrlichia; morula.

\section{Introduction}

Ehrlichiosis is becoming a growing problem in the USA. Although the majority of cases are self-resolving, in rare cases the infection may prove fatal. The number of ehrlichiosis cases due to Ehrlichia chaffeensis reported to the Centers for Disease Control and Prevention (CDC) since the disease became reportable in 1999 have increased steadily, from 200 cases in 2000 to 961 cases in 2008. In 2012, the case fatality rate, as reported by the CDC, was $1.1 \%$. There may be great difficulty in making a final diagnosis of infection if the suspicion for arthropodrelated illness is low.

\section{Case report 1}

A 56-year-old woman initially presented to an outlying hospital in Kentucky, USA, in June 2009 with complaints of persistent fevers and general malaise. Upon admission, she was pancytopenic and neutropenic (Table 1) and febrile $\left(101.1{ }^{\circ} \mathrm{F}\right)$. Her past medical history was significant for morbid obesity, sleep apnoea, hypertension, chronic venous insufficiency, migraine headaches, renal calculi and depression. Prior to her admission to an outlying hospital, she reported having a bronchitis-like illness and was treated by her family physician with trimethoprim/ sulfamethoxazole. The illness progressed to abdominal pain, vomiting and nausea, as well as a subjective elevated temperature and general malaise. Due to the patient's obesity and elevated D-dimer, a CT scan of her chest was attempted at the outlying hospital in order to rule out a pulmonary embolism. However the patient's size precluded an accurate assessment. A venous Doppler examination of the lower extremities was negative for deep-vein thrombosis. Following admission, the patient's condition progressively worsened. Sepsis from an unknown source was suspected; however, blood cultures were negative. The patient was initially placed on empiric antibiotic treatment, but despite treatment her condition worsened. During this time, she had increasing abdominal pain, hypotension, acute respiratory failure and progressive deterioration of hepatic and renal function. On the fifth day of her admission, she became increasingly confused, hypotensive and hypoxic, and developed a marked acidosis. As a result, she was quickly intubated and a transfer was arranged to the regional tertiary care center. She also displayed elevated liver and renal function values (Table 1). Her peripheral smear was notable for large and decreased numbers of platelets and acanthocytes, and slight toxic granulation of neutrophils. The patient was placed on broad-spectrum antibiotic, antifungal and antiviral agents. An abdominal exploratory laparoscopic surgery was performed due to the inability to obtain radiographic studies, but the procedure did not reveal any diagnostic 
Table 1. Selected laboratory results for the Case 1 patient, indicating severe cardiac, hepatic and renal dysfunction

\begin{tabular}{|lll|}
\hline Test & Result & Reference range \\
\hline Troponin I & $37.6 \mathrm{ng} \mathrm{ml}^{-1}$ & $0.0-0.034 \mathrm{ng} \mathrm{ml}^{-1}$ \\
Creatine kinase-MB & $13.1 \mathrm{ng} \mathrm{ml}^{-1}$ & $0.0-3.2 \mathrm{mg} \mathrm{ml}^{-1}$ \\
Aspartate aminotransferase & $327 \mathrm{IU} \mathrm{l}^{-1}$ & $15-50 \mathrm{IU} \mathrm{l}^{-1}$ \\
Alanine aminotransferase & $80 \mathrm{IU} \mathrm{l}^{-1}$ & $10-40 \mathrm{IU} \mathrm{l}^{-1}$ \\
Creatinine & $1.8 \mathrm{mg} \mathrm{dl}^{-1}$ & $0.5-1.2 \mathrm{mg} \mathrm{dl}^{-1}$ \\
Blood urea nitrogen & $42 \mathrm{mg} \mathrm{dl}^{-1}$ & $7-20 \mathrm{mg} \mathrm{dl}^{-1}$ \\
D-dimer & $5.4 \mathrm{mg} \mathrm{l}^{-1}$ & $0.54-2.09 \mathrm{mg} \mathrm{l}^{-1}$ \\
White blood cells & $14600 \mu \mathrm{l}^{-1}$ & $4800-10800 \mu \mathrm{l}^{-1}$ \\
Absolute granulocyte count & $12550 \mu \mathrm{l}^{-1}$ & $2000-8000 \mu \mathrm{l}^{-1}$ \\
Platelets & $60000 \mu \mathrm{l}^{-1}$ & $140000-370000 \mu \mathrm{l}^{-1}$ \\
\hline
\end{tabular}

findings. The next day, she experienced a seizure, with a temperature elevation to $103.3{ }^{\circ} \mathrm{F}$. Meningitis and cerebral infarct were considered possible aetiologies for her condition. An electrocardiogram revealed an ST elevation that was consistent with a myocardial infarction, and a subsequent echocardiogram revealed mild ventricular hypertrophy. Over the next 2 days, she became unresponsive and was determined as having hepatic and renal failure. Coagulation studies displayed a consumptive coagulopathy, suggestive of disseminated intravascular coagulation (DIC). A decision was made to withdraw care and the patient died. Permission for a complete autopsy and informed consent for research was granted from the family.

\section{Investigations}

The skin of the chest was remarkable for multiple confluent petechiae and ecchymoses. Marked oedema and jaundice were also present on the upper torso and the bilateral upper extremities. Gross examination of the cavities and organs revealed an enlarged liver, which displayed severe fatty infiltration. The pulmonary system displayed congestion and oedema of the left and right lungs. The brain displayed multiple punctuate petechiae that were evident in the frontal, temporal and parietal lobes, as well as in the hypothalamus and basal ganglia. Diffuse congestion with oedema was present throughout the cerebrum.

A microscopic examination of the spleen showed multiple microthrombi with infarcts and congestion. Both the right and left kidneys displayed global infarction, scant interstitial inflammation, thinned cortex, congestion and tubular necrosis. The brain was remarkable for gliosis, congestion, perivascular lymphocytic infiltrates, microglial nodules and Alzheimer type II astrocytes, which were present diffusely throughout the cerebrum, consistent with liver failure and end-organ dysfunction. The liver revealed marked global steatosis, as well as pericentral vein ischaemia. The bone marrow was hypercellular (70\% cellularity) with increased absolute megakaryocytes and decreased mature neutrophils, consistent with peripheral consumption of platelets and neutrophils. Ancillary studies for suspected tick-borne aetiologies included serological analysis, peripheral smear review and DNA analysis. The serum was sent for ehrlichiosis testing the day before the patient expired, and she was immediately started on doxycycline before the results were known. Serology was reported at 1 day post-mortem as positive for IgM and IgG for both Ehrlichia and Anaplasma. The serological results were negative for Rickettsia typhi and Borrelia burgdorferi. Cross-reaction of Ehrlichia and Anaplasma antibodies is common, and therefore a more definite result was not possible from serology alone. Subsequently, a retrospective review of the patient's peripheral blood smears revealed rare cytoplasmic morulae in both neutrophils and monocytes, consistent with an Ehrlichia infection (Fig. 1).

\section{Diagnosis}

As a result of the serological findings and peripheral smear review, tissue blocks of the heart, bone marrow and spleen were sent to the CDC for Ehrlichia confirmation by immunohistochemical and PCR analysis. Immunohistochemistry using polyclonal antibodies for

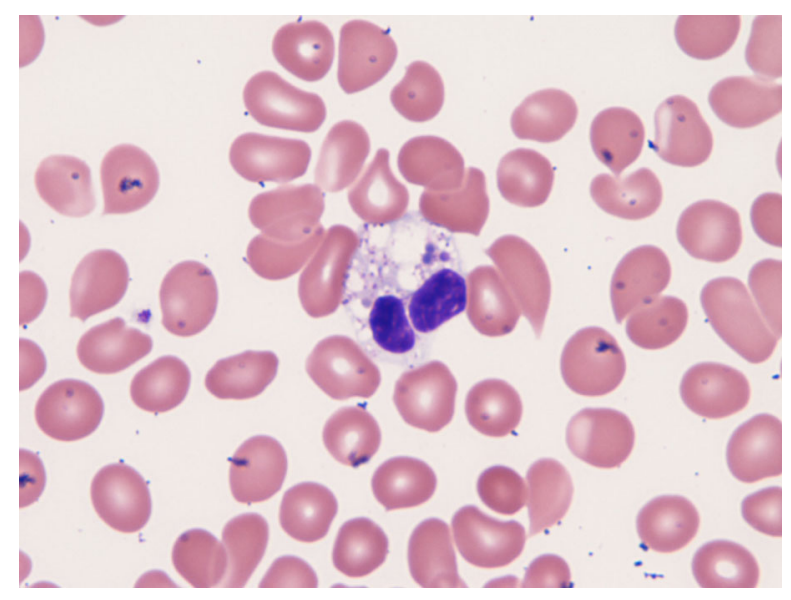

Fig. 1. Peripheral smear displaying multiple morulae inside an apoptotic neutrophil. The morulae are composed of Ehrlichia organisms. 
Anaplasma and Ehrlichia species was performed on all three blocks submitted. All blocks were negative for Anaplasma. However, the spleen tissue was positive for Ehrlichia species, with frequent intracellular staining. PCR analysis of the heart tissue block was positive for Ehrlichia, whilst the spleen and bone-marrow blocks were negative. The CDC stated that the organisms in the heart tissue contained $100 \%$ nucleotide sequence similarly to the homologous fragment of E. chaffeensis. As a result, the cause of death was listed as 'Ehrlichiosis with overwhelming sepsis.'

\section{Case report 2}

A few weeks following Case 1, a 45-year-old Kentucky man was found unconscious and unresponsive by emergency medical services with no prior reported illness. The patient was initially seen at an outlying hospital, where he arrived in status epilepticus. He was febrile $\left(102.3^{\circ} \mathrm{F}\right)$, and laboratory tests revealed a marked pancytopenia along with renal and liver failure. An electrocardiogram displayed evidence of an acute myocardial infarction, which was supported by a greatly elevated troponin I level (Table 2). He was transferred unresponsive to the regional tertiary care center. Upon admission, a fine petechial rash was noted on the bilateral lower extremities. Coagulation studies revealed a consumptive coagulopathy, suggesting DIC. A peripheral blood smear revealed anisocytosis with occasional target cells, burr cells and schistocytes. Hepatic transaminases were significantly elevated, renal function was adversely affected and the patient had metabolic acidosis (Table 1). He was treated for sepsis, DIC and possible meningitis early in the course of disease. After admission, the patient's condition continued to deteriorate rapidly. His prognosis was discussed with the family and the decision to withdraw care was made. He died the next morning, and the patient's family granted permission for a complete autopsy and informed consent for research.

\section{Investigations}

Confluent reddish-purple petichiae were present on the skin of the forehead, nose, upper anterior torso and bilateral lower extremities. Gross examination of the organs and cavities revealed severely congested lungs. Microscopic examination was notable for diffuse conges-

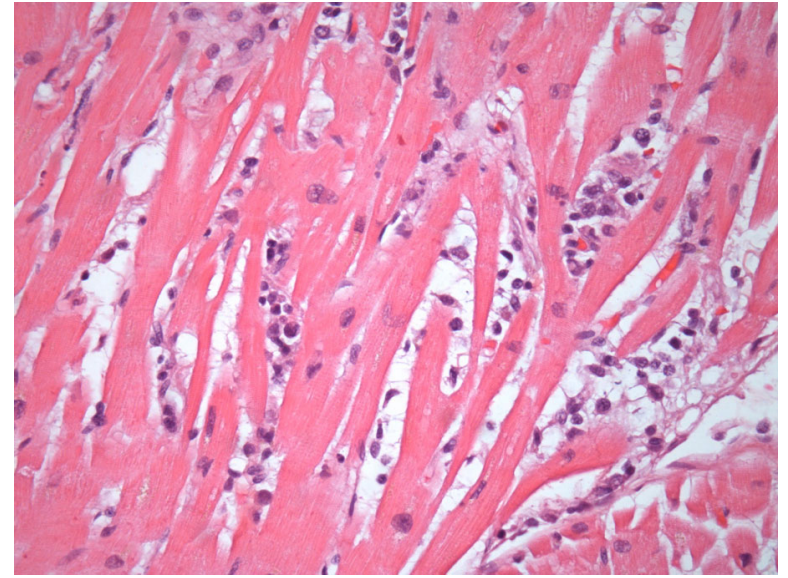

Fig. 2. Myocardial photomicrograph displaying diffuse lymphocytic infiltration with apoptosis of the myocytes (left).

tion and consolidation of the lungs with concomitant chronic and acute inflammation, haemorrhage, fibrin deposition and oedema. The myocardium showed a lymphocytic infiltration with myonecrosis, focal fibrosis and nucleomegaly (Fig. 2). The cerebrum and cerebellum displayed migroglial nodules, perivascular lymphocytic infiltrates and lymphocytic leptomeningitis. In addition, the cerebrum displayed evidence of diffuse oedema.

After comparing the similarities to Case 1, we suspected that E. chaffeensis might also be the case of death in this patient. As a result, we sent post-mortem tissue blocks from representative sections of the kidney and hippocampus, as well as the intraventricular septum and the right ventricle of the heart, to the CDC for investigation of a tick-borne aetiology. The CDC performed immunohistochemistry on all four tissue blocks for spotted fever group rickettsia, typhus group rickettsia and Ehrlichia species (polyclonal antibody reactive with Ehrlichia canis, E. chaffeensis and Ehrlichia ewingii). All four blocks were positive for Ehrlichia species and negative for spotted fever and typhus group rickettsia. PCR was subsequently performed for species identification and was positive for the E. chaffeensis $16 \mathrm{~S}$ rRNA gene.

Table 2. Selected laboratory results for the Case 2 patient indicating severe cardiac, hepatic, and renal dysfunction

\begin{tabular}{|lll|}
\hline Test & Result & Reference range \\
\hline Troponin I & $>80.000 \mathrm{ng} \mathrm{ml}^{-1}$ & $0.00-0.40 \mathrm{ng} \mathrm{ml}^{-1}$ \\
Creatine kinase & $94,880 \mathrm{IU} \mathrm{l}^{-1}$ & $30-135 \mathrm{IU} \mathrm{l}^{-1}$ \\
Aspartate aminotransferase & $750 \mathrm{IU} \mathrm{l}^{-1}$ & $10-50 \mathrm{IU} \mathrm{l}^{-1}$ \\
Alanine aminotransferase & $117 \mathrm{IU} \mathrm{l} l^{-1}$ & $20-70 \mathrm{IU} \mathrm{l^{-1 }}$ \\
Creatinine & $2.5 \mathrm{mg} \mathrm{l}^{-1}$ & $0.7-1.4 \mathrm{mg}^{-1}$ \\
Blood urea nitrogen & $78 \mathrm{mg} \mathrm{l}^{-1}$ & $7-20 \mathrm{mg} \mathrm{l}^{-1}$ \\
D-dimer & $15.1 \mathrm{mg} \mathrm{l}^{-1}$ & $0.0-1.6 \mathrm{mg} \mathrm{l}^{-1}$ \\
Platelets & $6000 \mu l^{-1}$ & $140000-370000 \mu l^{-1}$ \\
\hline
\end{tabular}




\section{Diagnosis}

As a result, the CDC determined that the meningoencephalitis, myocarditis and interstitial nephritis displayed immunochemical and molecular evidence of infection with E. chaffeensis. This finding was in general agreement with our original cause of death as lymphocytic myocarditis, presumed due to viral illness, in conjunction with lymphocytic encephalitis and leptomeningitis'. Upon further review, the cause of death was determined to be related to E. chaffeensis infection.

\section{Discussion}

Human monocytic ehrlichiosis (HME) is caused by infection of monocytes by E. chaffeensis, an obligate intracellular Gram-negative bacteria. The reservoir is predominantly the white-tailed deer (Odocoileus virginanus) (Ganguly and Mukhopadhayay, 2008; Ismail et al., 2010). The primary tick vector of HME is the lone star tick (Amblyomma americanum), which is geographically distributed in southeast, south central and mid-west USA (Ganguly and Mukhopadhayay, 2008). HME was first described in 1986, and more than 2300 cases have been reported to the CDC during the past two decades (Ismail et al., 2010; Dahlgren et al., 2011). Of 1206 nationwide cases documented by the CDC from 2000 to 2007, $49 \%$ of patients required hospitalization for HME, with 19 fatal cases $(1.9 \%)$ reported during the same time period. Nationally, the incidence of HME has increased from 0.8 cases per million of the population in 2000 to 3.0 cases per million in 2007 (Dahlgren et al., 2011). Ehrlichia species consist of a group of aetiologically and epidemiologically distinct entities that have a similar clinical presentation. Anaplasma phagocytophilum, E. ewingii and E. chaffeensis all belong to the Anaplasmataceae family, and have been implicated in the overall clinical picture termed 'ehrlichiosis' (Ganguly and Mukhopadhayay, 2008; Dahlgren et al., 2011).

Most E. chaffeensis (HME) transmission occurs during the summer months, with the peak period of May-July corresponding to the lone star tick's preferred feeding time (Ismail et al., 2010; Dahlgren et al., 2011; Schneider, 2009). Symptoms of HME often include fever, headache, malaise and myalgia (Ismail et al., 2010; Dahlgren et al., 2011; Schneider, 2009; Pavelites and Prahlow, 2011) Haematological perturbations include leukopenia, anaemia and thrombocytopenia. Elevations of serum hepatic transaminases are common (Sehdev and Dumler, 2003). Symptoms usually begin a median of 9 days following a tick bite, and the majority of patients will seek medical help within 4 days of the onset of the illness (Ganguly and Mukhopadhayay, 2008; Ismail et al., 2010).

Altered mental status is reported in up to $20 \%$ of patients, with symptoms of meningeal irritation such as nuchal rigidity, photophobia and severe headaches present in nearly $10 \%$ of patients with HME. Cerebral spinal fluid will show abnormal findings in $60 \%$ of cases with altered mentation. Most cases have a lymphocytic pleocytosis, but one-third of patients will have a neutrophilic predominance (Hongo and Bloch, 2006). In both of our cases, the patients presented with fever, malaise, haematological abnormalities and elevation of serum hepatic transaminases. Laboratory studies and autopsy tissue examination revealed the development of DIC in both patients. This coagulopathy resulted in petechial rash, elevated $\mathrm{D}$-dimer, thrombocytopenia and multi-organ infarction. The commonly described symptoms of HME may be related to DIC as a frequent complication of HME. Demonstrating the organism in tissue by immunohistochemistry or molecular studies in immunocompetent patients may be difficult, as few organisms are present. Although the time frame from the onset of symptoms to death was rapid in the first case and prolonged in the second case, the clinical progression was similar for both, with seizures, clinically suspected myocardial infarction, mental status changes, and concomitant hepatic and renal failure. The clinical team investigated for the same differential diagnoses in both cases, but the possibility of a tick-borne illness was not raised in either patient until late in the presentation or after death. After diagnosing an Ehrlichia infection in our first case, we compared the findings with the second case. Originally, the cause of death in the second case was attributed to 'lymphocytic myocarditis, presumed due to viral illness, in conjunction with lymphocytic encephalitis and leptomeningitis,' which was consistent with the clinical presentation as well as our autopsy findings. However, because of our recent diagnosis of ehrlichiosis, and also due to the case presenting at the peak time for $E$. chaffeensis infection in Kentucky, we decided to send our blocks to the CDC for further analysis. When the CDC report confirmed the presence of Ehrlichia in our second case, it highlighted the need to have a high degree of suspicion when encountering these tick-borne illnesses. It is highly unlikely that we would have been able to determine E. chaffeensis as the cause of death in our second case without the diagnosis in our first case. Suspicion of HME early in the clinical presentation is paramount. Ehrlichiosis, as well as other rickettsial diseases, can cause significant morbidity and mortality if they are not suspected and treated in a timely manner (Prince et al., 2007). In the south-east, south central and mid-western USA, ehrlichiosis should be placed high in the differential diagnosis along with other tick-borne aetiologies, especially during the peak summer months (Prince et al., 2007; Seddighzadeh et al., 2009). Although serological testing and a peripheral smear review should be ordered if HME is suspected, clinicians should not wait for test results, as serological testing may be negative for up to 3 weeks after the onset of symptoms (Prince et al., 2007). The CDC recommends examination of paired serum samples obtained 2-3 weeks apart as the most effective approach to confirm HME serology. A positive HME result is defined as a fourfold or greater increase in either IgM or IgG serology between paired samples (Dahlgren et al., 2011; Prince et al., 2007). Peripheral 
smear analysis alone also poses problems, as morulae are identified in neutophils or monocytes in only $20 \%$ of cases.

Treatment therefore should not be withheld while waiting for the results of diagnostic tests. HME can be treated effectively with a regimen of doxycycline (100 mg orally or intravenously for 10-14 days), provided the diagnosis is made in a timely manner (Ismail et al., 2010). Of note, both patients were taking trimethoprim/sulfamethoxazole when infected by E. chaffeensis. It has been noted in both adult and paediatric populations that treatment with sulfonamide antibiotics, such as trimethoprim/sulfamethoxazole, is associated with more severe manifestations and outcomes (Ismail et al., 2010; Peters et al., 2000). Whether this finding represents an actual causal relationship or is the result of delayed treatment with doxycycline is currently unknown (Ismail et al., 2010). As the prevalence of HME has increased, so too has the interest in discovering the molecular machinery behind this emerging pathogen. Proteonomic analysis of E. chaffeensis has revealed 1021 proteins, nearly all of which have functions in infected human hosts regarding metabolism, pathogenesis and regulation (Lin et al., 2011). In addition, other recent studies have explored the role of natural killer cells promoting tissue injury in fatal E. chaffeensis cases (Stevenson et al., 2010). In addition, the role of effector molecules in blocking the anti-ehrlichial activity of IFN- $\gamma$ has been established (Wakeel et al., 2010). Although there is still more work to be done, new insights into the mechanisms of ehrlichial infection are emerging. E. chaffeensis generally has a benign course in most individuals. Ehrlichia infection may range from a mild illness to death if not adequately treated in a timely manner. Therefore, with the increasing prevalence of Ehrlichia across the USA, it is paramount to include this tick-borne illness in the differential diagnosis.

\section{Acknowledgements}

There are no conflicts of interests between the authors and any aspect of this case.

\section{References}

Dahlgren, F. S., Mandel, E. J., Krebs, J. W., Massung, R. F. \& McQuiston, J. H. (2011). Increasing incidence of Ehrlichia chaffeensis and Anaplasma phagocytophilum in the United States, 2000-2007. Am J Trop Med Hyg 85, 124-131.

Ganguly, S. \& Mukhopadhayay, S. K. (2008). Tick-borne ehrlichiosis infection in human beings. J Vector Borne Dis 45, 273-280.

Hongo, I. \& Bloch, K. C. (2006). Ehrlichia infection of the central nervous system. Curr Treat Options Neurol 8, 179-184.

Ismail, N., Bloch, K. \& McBride, J. (2010). Human ehrlichiosis and anaplasmosis. Clin Lab Med 30, 261-292.

Lin, M., Kikuchi, T., Brewer, H. M., Norbeck, A. D. \& Rikihisa, Y. (2011). Global proteonomic analysis of two tick-borne emergine zoonotic agents: Anaplasma phagocytophilum and Ehrlichia chaffeensis. Front Microbio 2, 24.

Pavelites, J. J. \& Prahlow, J. A. (2011). Fatal human monocytic ehrlichiosis: a case study. Forensic Sci Med Pathol 7, 287-293.

Peters, T. R., Edwards, K. M. \& Standaert, S. M. (2000). Severe ehrlichiosis in an adolescent taking trimethoprim-sulfamethoxazole. Pediatr Infect Dis J 19, 170-172.

Prince, L. K., Shah, A. A., Martinez, L. J. \& Moran, K. A. (2007). Ehrlichiosis: making the diagnosis in the acute setting. South Med J 100, 825-828.

Schneider, J. G. (2009). Human ehrlichiosis: a case study. Clin Lab Sci 22, 3-8.

Seddighzadeh, A., Pinger, R. R., Zercher, A., Steiner, F. E., Klyachko, O., Vann, C. N., Clay, K. \& Fuqua, C. (2009). Strains of Ehrlichia chaffeensis in southern Indiana, Kentucky, Mississippi, and North Carolina. J Med Entomol 46, 1468-1473.

Sehdev, A. E. \& Dumler, J. S. (2003). Hepatic pathology in human monocytic ehrlichiosis. Am J Clin Pathol 119, 859-865.

Stevenson, H. L., Estes, M. D., Thirumalapura, N. R., Walker, D. H. \& Ismail, N. (2010). Natural killer cells promote tissue injury and systemic inflammatory responses during fatal Ehrlichia-induced toxic shock-like syndrome. Am J Pathol 177, 766-776.

Wakeel, A., Zhu, B., Yu, X. J. \& McBride, J. W. (2010). New insights into molecular Ehrlichia chaffeensis-host interactions. Microbes Infect 12, 337-345. 